\title{
${ }^{18} \mathrm{~F}-\mathrm{FDG}-\mathrm{PET} / \mathrm{CT}$ and diffusion-weighted MRI for monitoring a BRAF and CDK 4/6 inhibitor combination therapy in a murine model of human melanoma
}

Ralf S. Eschbach ${ }^{1 *}$, Philipp M. Kazmierczak', Maurice M. Heimer ${ }^{1}$, Andrei Todica², Heidrun Hirner-Eppeneder ${ }^{1}$, Moritz J. Schneider ${ }^{1,3}$, Georg Keinrath ${ }^{1}$, Olga Solyanik', Jessica Olivier², Wolfgang G. Kunz ${ }^{1}$, Maximilian F. Reiser ${ }^{1}$, Peter Bartenstein ${ }^{2}$, Jens Ricke ${ }^{1}$ and Clemens C. Cyran ${ }^{1}$

\begin{abstract}
Background: The purpose of the study was to investigate a novel BRAF and CDK 4/6 inhibitor combination therapy in a murine model of BRAF-V600-mutant human melanoma monitored by ${ }^{18} \mathrm{~F}-\mathrm{FDG}-\mathrm{PET} / \mathrm{CT}$ and diffusionweighted MRI (DW-MRI).

Methods: Human BRAF-V600-mutant melanoma (A375) xenograft-bearing balb/c nude mice $(n=21)$ were imaged by ${ }^{18} \mathrm{~F}-\mathrm{FDG}$-PET/CT and DW-MRI before (day 0 ) and after (day 7) a 1-week BRAF and CDK 4/6 inhibitor combination therapy ( $n=12$; dabrafenib, $20 \mathrm{mg} / \mathrm{kg} / \mathrm{d}$; ribociclib, $100 \mathrm{mg} / \mathrm{kg} / \mathrm{d}$ ) or placebo $(n=9)$. Animals were scanned on a small animal PET after intravenous administration of $20 \mathrm{MBq}{ }^{18} \mathrm{~F}-\mathrm{FDG}$. Tumor glucose uptake was calculated as the tumor-to-liver-ratio (TTL). Unenhanced $C T$ data sets were subsequently acquired for anatomic coregistration. Tumor diffusivity was assessed by DW-MRI using the apparent diffusion coefficient (ADC). Anti-tumor therapy effects were assessed by ex vivo immunohistochemistry for validation purposes (microvascular density - CD31; tumor cell proliferation - Ki-67).
\end{abstract}

Results: Tumor glucose uptake was significantly suppressed under therapy $\left(\Delta T \mathrm{~L}_{\text {Therapy }}-1.00 \pm 0.53 \mathrm{vs}\right.$. $\Delta T \mathrm{~L}_{\text {Control }}$ $0.85 \pm 1.21 ; p<0.001$ ). In addition, tumor diffusivity was significantly elevated following the BRAF and CDK $4 / 6$ inhibitor combination therapy $\left(\triangle A D C_{\text {Therapy }} 0.12 \pm 0.14 \times 10^{-3} \mathrm{~mm}^{2} / \mathrm{s} ; \triangle A D C_{\text {Control }}-0.12 \pm 0.06 \times 10^{-3} \mathrm{~mm}^{2} / \mathrm{s} ; p<0.001\right.$ ). Immunohistochemistry revealed a significant suppression of microvascular density (CD31, $147 \pm 48$ vs. $287 \pm 92$; $p=0.001)$ and proliferation (Ki-67, $3718 \pm 998$ vs. $5389 \pm 1332 ; p=0.007$ ) in the therapy compared to the control group.

Conclusion: A novel BRAF and CDK 4/6 inhibitor combination therapy exhibited significant anti-angiogenic and anti-proliferative effects in experimental human melanomas, monitored by ${ }^{18} \mathrm{~F}-\mathrm{FDG}-\mathrm{PET} / \mathrm{CT}$ and DW-MRI.

Keywords: Melanoma, BRAF inhibitor, CDK inhibitor, ${ }^{18}$ F-FDG-PET, Diffusion-weighted MRI, Therapy monitoring

\footnotetext{
* Correspondence: ralf.eschbach@med.Imu.de

Ralf S. Eschbach and Philipp M. Kazmierczak contributed equally to the project.Ralf S. Eschbach and Philipp M. Kazmierczak share first authorship. 'Department of Radiology, Laboratory for Experimental Radiology, University Hospital, Ludwig-Maximilians-University Munich, Marchioninistr. 15, 81377 München, Germany

Full list of author information is available at the end of the article
} 


\section{Background}

The mitogen-activated protein kinase (MAPK) pathway controls cell cycle progression, survival, and proliferation in human cells $[1,2]$. B-rapidly accelerated fibrosarcoma (BRAF) gene mutations $\mathrm{V} 600 \mathrm{E} / \mathrm{K}$ were identified as key drivers of oncogenesis in melanoma, as they lead to overactivation of the MAPK pathway and uncontrolled cell proliferation [2]. Thus, selective inhibition of the BRAF gene emerged as a novel, targeted treatment regimen in advanced or unresectable melanoma [3, 4]. However, tumor response to BRAF inhibitor monotherapy may be limited by intrinsic or acquired resistance [5-7]. Consequently, combination therapies of BRAF inhibitors and additional therapeutics potentially overcoming resistance were investigated in recent years $[5,8]$. One possible mechanism of acquired BRAF inhibitor resistance is MAPK pathway activation via the mitogenactivated extracellular signal-regulated kinase (MEK). Co-targeting the MAPK pathway by a BRAF and MEK inhibitor combination therapy yields high response rates as well as prolonged overall and progression-free survival in advanced BRAF-mutant melanoma compared to other available treatment strategies, including BRAF inhibitor monotherapy [9]. A possible mechanism of intrinsic BRAF inhibitor resistance is cyclin D1 overexpression with cyclin-dependent kinase (CDK) 4 mutation/amplification $[2,5]$. Cyclin D1 promotes cell cycle progression by binding to CDK 4 and 6 , which act as downstream mediators of the MAPK pathway and regulate the cell cycle via the retinoblastoma tumor suppressor protein $[2,5]$. Yadav et al. recently demonstrated that the selective inhibition of CDK 4/6 leads to tumor growth regression in a BRAF inhibitor-resistant in vivo model of human melanoma (A375) [10]. In 2017, based on the results of the MONALEESA-2 trial, the U.S. Food and Drug Administration approved the CDK 4/6 inhibitor ribociclib combined with an aromatase inhibitor for the treatment of advanced or metastatic, hormone receptor-positive and human epidermal growth factor receptor 2-negative breast cancer in postmenopausal women [11]. The addition of a CDK $4 / 6$ inhibitor to BRAF inhibitor monotherapy represents a novel strategy to overcome cyclin D1-dependent resistance. Dual inhibition of the MAPK pathway by a BRAF and CDK 4/6 inhibitor combination therapy may thus be a promising future therapy regimen in advanced melanoma.

Imaging plays a central role for the non-invasive tumor response assessment in clinical oncology. Morphologybased criteria of tumor response, e. g., RECIST (Response Evaluation Criteria in Solid Tumors), provide a valuable clinical tool to differentiate between partial/complete response, progressive, and stable disease [12]. These criteria are based on the number and size of tumor manifestations, which are commonly assessed by morphological imaging modalities such as computed tomography (CT) or magnetic resonance imaging (MRI). However, in contrast to traditional, primarily cytotoxic therapies, novel targeted therapies exhibit only subtle effects on tumor size [13]. Thus, morphology-based tumor response criteria are of only limited applicability in targeted therapy regimens [14]. Functional and molecular imaging modalities allow for a non-invasive tumor characterization beyond morphology, delivering information on tumor pathophysiology such as tumor glucose metabolism $\left({ }^{18} \mathrm{~F}\right.$-fluorodeoxyglucose positron emission tomography; ${ }^{18} \mathrm{~F}$-FDG-PET) and tumor cellularity (diffusion-weighted MRI; DWMRI). Both ${ }^{18}$ F-FDG-PET and DW-MRI demonstrated their potential to generate non-invasive imaging biomarkers of therapy response in melanoma under targeted therapy [15-18].

As a proof of principle, the present study is a first approach to explore a selective CDK $4 / 6$ inhibitor as novel combination compound for dual inhibition of the MAPK signal pathway in melanoma therapy. The aim of this experimental study was to close this gap of knowledge, evaluating a novel BRAF and CDK 4/6 inhibitor combination therapy in a murine model of human BRAF-V600mutant melanoma using a multimodal imaging protocol of ${ }^{18} \mathrm{~F}$-FDG-PET/CT and DW-MRI. We hypothesized that a BRAF and CDK 4/6 inhibitor combination therapy exhibits significant anti-angiogenic and anti-proliferative effects in experimental human melanomas in mice and that the according alterations in tumor pathophysiology can be non-invasively monitored by ${ }^{18} \mathrm{~F}-\mathrm{FDG}$ $\mathrm{PET} / \mathrm{CT}$ and DW-MRI in vivo validated by ex vivo immunohistochemistry.

\section{Methods}

The experiments were performed in accordance with the Guidelines for the Care and Use of Laboratory Animals of the National Institutes of Health and with approval by the Government Committee for Animal Research.

\section{Animal model and experimental protocol}

After diluting human melanoma cells (A375, ATCC CRL-1619 ${ }^{\text {max }}$, CLS Cell Lines Service GmbH, Eppelheim, Germany) in a total volume of $0.1 \mathrm{~mL}$ as a 1:1 solution of phosphate buffered saline (PBS pH 7.4; GIBCO Life Technologies, Darmstadt, Germany) and Matrigel $^{\mathrm{mu}}$ (BD Biosciences, San Jose, CA), $3 \times 10^{6}$ cells per mouse were injected subcutaneously into the left abdominal flank of $n=21$ athymic balb/c nude mice (Charles River, Sulzfeld, Germany). When tumors reached a diameter of $0.5 \mathrm{~cm}$, animals were randomly assigned to either the therapy $(n=12)$ or the control group $(n=9)$. Imaging was performed on day 0 (baseline) and day 7 (follow-up) using a multimodal imaging protocol of ${ }^{18} \mathrm{~F}-\mathrm{FDG}$ PET/CT and DW-MRI. Imaging was performed under inhalation 
anesthesia $\left(2.5 \%\right.$ in $1.0 \mathrm{~L} 100 \% \mathrm{O}_{2} / \mathrm{min}$ for induction, $1.5 \%$ in $1.0 \mathrm{~L} 100 \% \mathrm{O}_{2} / \mathrm{min}$ for maintenance). Subsequently to the baseline scan, animals were treated daily with either a combination of BRAF inhibitor dabrafenib (20 mg/kg/day; Novartis AG, Basel, Switzerland) and CDK4/6 inhibitor ribociclib $(100 \mathrm{mg} / \mathrm{kg} /$ day; Novartis AG, Basel, Switzerland) in the therapy group or a volume-equivalent placebo solution $(0.5 \%$ hydroxymethyl cellulose and $0.2 \%$ Tween- 80 in $\mathrm{ddH}_{2} 0$ ) in the control group. Immediately after follow-up imaging on day 7 , animals were sacrificed and tumors were explanted and fixed in formalin for immunohistochemical analysis with regard to tumor microvascular density (CD31) and tumor cell proliferation (Ki-67). Figure 1 provides an overview of the experimental protocol.

\section{PET imaging}

Small-animal PET was performed on a preclinical PET scanner (Inveon $\mu \mathrm{PET}$, Siemens Healthineers, Erlangen, Germany). For hygienic reasons and allowing regulated anesthesia delivery, mice were placed inside a custombuilt acrylic glass-imaging chamber in prone position. $45 \mathrm{~min}$ after manual intravenous injection of $20 \mathrm{MBq}$ $(\sim 100 \mu \mathrm{L}){ }^{18} \mathrm{~F}-\mathrm{FDG}$ per animal, a transmission scan (15 min) was acquired for scatter and attenuation correction. Subsequently, PET list-mode data acquisition was initiated from 60 to $90 \mathrm{~min}$ (30 min emission).

All PET data were post-processed on an Inveon Acquisition Workplace (Siemens Healthineers, Erlangen, Germany). Images were reconstructed as a static image using OSEM 3D (4 iterations) and MAP 3D (32 iterations) algorithm in a $256 \times 256$ matrix with a zoom value of $100 \%$ as described previously [19]. To calculate the metabolic tumor volume (MTV), a PET/CT-guided volume of interest (VOI) surrounding the tumor was drawn manually and a threshold-value of $30 \%$ of the hottest voxels was applied. Background was determined as mean uptake in a $9 \mathrm{~mm}^{3}$ VOI in the right liver lobe. Tumor-to-liverratio (TTL, VOImaxtumor/VOImeanliver) was determined as a semiquantitative measurement of tumor radiotracer accumulation before (day 0) and after treatment (day 7). Figure 2 demonstrates the process of PET/CT VOI selection.

After PET measurements, additional CT images were acquired on a latest generation clinical CT (SOMATOM Force, Siemens Healthineers, Erlangen, Germany), maintaining the animals in the same position within the acrylic imaging chamber for morphological correlation, as described previously [19].

\section{MRI}

MRI measurements were conducted on a clinical 3 Tesla scanner (MAGNETOM Skyra, Siemens Healthineers, Erlangen, Germany) using a 16-channel wrist coil (Siemens Healthineers, Erlangen, Germany). Mice were positioned head-first in prone position. For the assessment of tumor morphology and tumor volume as morphology-based surrogate of tumor response, T2weighted MR images were acquired using a 2D Turbo Spin Echo sequence $(\mathrm{TR}=5470 \mathrm{~ms}, \mathrm{TE}=91 \mathrm{~ms}$, inplane resolution $0.3 \times 0.3 \mathrm{~mm}$, matrix size $192 \times 192$, slice thickness $1.5 \mathrm{~mm}$ ). In addition, DW-MRI data sets were acquired using a single shot echo planar imaging sequence $(\mathrm{TR}=3200 \mathrm{~ms}, \mathrm{TE}=50 \mathrm{~ms}$, FOV $=84 \mathrm{~mm}$, acquisition matrix $=64 \times 64$, reconstructed matrix $=128 \times 128$, slice thickness $2 \mathrm{~mm}, 8$ averages, time to acquisition $180 \mathrm{~s} ; 3$ b-values: $\left.\mathrm{b}=0 \mathrm{~s} / \mathrm{mm}^{2} / 200 \mathrm{~s} / \mathrm{mm}^{2} / 800 \mathrm{~s} / \mathrm{mm}^{2}\right)$. Apparent diffusion coefficient (ADC) maps were calculated by voxel-wise least-squares fitting of the model

$$
\mathrm{S}(\mathrm{b})=\mathrm{S}_{0} \cdot \mathrm{e}^{-\mathrm{b} \cdot \mathrm{ADC}}
$$

MRI data sets were analyzed on an external workstation using a dedicated, in-house written post-processing software (PMI; Platform for Research in Medical Imaging, version 0.4) [20]. For each measurement, a multi-slice VOI was drawn manually over the entire tumor. The median value inside this VOI was used to calculate a representative tumor ADC value for statistical analysis.

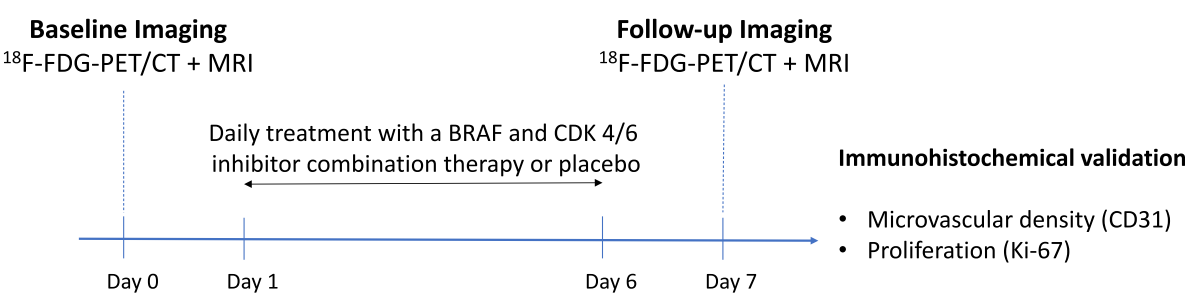

Fig. 1 Experimental protocol. ${ }^{18} \mathrm{~F}-\mathrm{FDG}-\mathrm{PET} / \mathrm{CT}$ and MRI baseline scans were performed on day 0. Animals received either a BRAF and CDK4/6 inhibitor combination therapy (therapy group) or a volume-equivalent placebo (control group) administered daily over the course of 6 days. Follow-up ${ }^{18}$ F-FDG-PET/CT and MRI scans were performed on day 7 . Subsequent to follow-up imaging, animals were sacrificed. The tumors were explanted to undergo the immunohistochemical analysis with regard to tumor microvascular density (CD31) and tumor cell proliferation (Ki-67) 


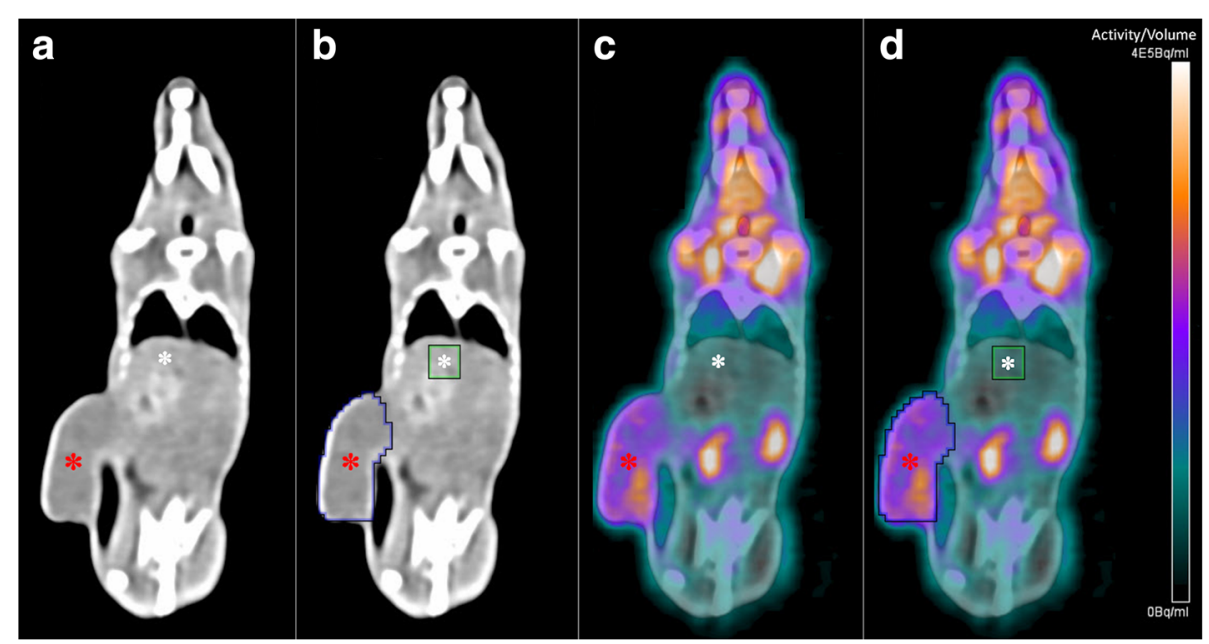

Fig. 2 PET/CT VOI Selection. a and b: coronal unenhanced CT without (a) and with (b) VOls. $\mathbf{c}$ and $\mathbf{d}$ : Fused coronal PET/CT data sets without (c) and with (d) VOls. Note the tumor in the lateral flank (red asterisk). The liver is indicated by a white asterisk. To calculate the metabolic tumor volume, a PET/CT-guided VOI surrounding the tumor was drawn. Background was determined in a VOI in the right liver lobe and the TTL was calculated accordingly

\section{Immunohistochemistry}

Three micrometer tissue sections were cut from the formalin-fixed and paraffin-embedded tumor tissue and stained with regard to microvascular density (CD31) and tumor cell proliferation (Ki-67). After de-waxing and rehydration following standard procedures (pre-heating at $60{ }^{\circ} \mathrm{C}$, washing in xylene substitute (Neo-Clear, Merck KgaA, Darmstadt, Germany) and rehydration in a graded series of ethanol $(100,95,80$, and $70 \%$, respectively) followed by double distilled water, antigen demasking was performed by microwave irradiation at $600 \mathrm{~W}$ in a $0.1 \mathrm{M}$ citrate buffer solution ( $\mathrm{pH} 6.0$ ). Tissue samples were subsequently antibody-incubated at $4{ }^{\circ} \mathrm{C}$ over night (antibodies: monoclonal rabbit anti-Ki-67 antibody; SP6, Abcam ab16667 1:100, Cambridge, United Kingdom; polyclonal rabbit anti-CD31 primary antibody; Abcam ab28364 1:50, Cambridge, United Kingdom).

According to the manufacturer's instructions, further work-up of tissue samples was performed using the EnVision + System HRP (DAB or AEC) (DAKO Diagnostika, Hamburg, Germany) kit. Slides were counterstained using Mayer's Haemalaun (Merck KgaA, Darmstadt, Germany) and covered with Kaiser's Glycerin Gelatine (Merck KgaA, Darmstadt, Germany). Results were quantified as the number of positively stained nuclei (Ki-67) or positively stained microvessels (CD31) in ten random fields at $200 \times$ magnification.

\section{Statistical analysis}

In this randomized placebo-controlled preclinical trial the statistical analyses were performed using commercially available statistics software (SPSS 23, IBM Corp., Armonk, NY). For intergroup comparisons of the imaging and the immunohistochemical parameters, the Mann-Whitney-U-test was applied. For intragroup comparisons between baseline (day 0) and follow-up (day 7), a Wilcoxon signed-rank test was performed. Correlations between the imaging and the immunohistochemical parameters were assessed by Spearman's correlation coefficient. Continuous variables were presented as means with standard deviations. The confirmatory tests were performed against a significance level $\alpha=0.05$ with Bonferroni corrections.

\section{Results}

The experimental protocol was successfully completed in $n=21$ animals. There were no significant $(p>0.05)$ intergroup differences between therapy and control

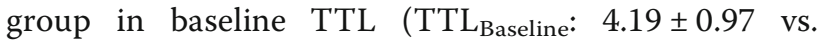
$3.70 \pm 0.90 ; p=0.193)$ and baseline ADC (ADC Baseline: $0.78 \pm 0.10 \times 10^{-3} \mathrm{~mm}^{2} / \mathrm{s}$ vs. $0.79 \pm 0.10 \times 10^{-3} \mathrm{~mm}^{2} / \mathrm{s}$ $p=0.754)$ as well as in metabolic $\left(161.1 \pm 86.5 \mathrm{~mm}^{3}\right.$ vs. $\left.217.9 \pm 145.8 \mathrm{~mm}^{3} ; p=0.464\right)$ and morphological $\left(94.6 \pm 71.4 \mathrm{~mm}^{3}\right.$ vs. $\left.134.3 \pm 89.0 \mathrm{~mm}^{3} ; p=0.219\right)$ tumor volumes.

\section{${ }^{18}$ F-FDG-PET/CT}

We observed a significant reduction of TTL under therapy $\left(\mathrm{TTL}_{\text {Therapy }}\right.$ from $4.19 \pm 0.97$ to $3.19 \pm 0.97$; $p=0.002$ ). In the control group, TTL demonstrated a non-significant increase from baseline to follow-up (TTL $_{\text {Control }}$ from $3.70 \pm 0.90$ to $4.55 \pm 0.91 ; p=0.14$ ). Follow-up TTL values were significantly lower in the

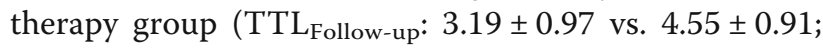
$p=0.007)$. Moreover, $\Delta \mathrm{TTL}\left(\mathrm{TTL}_{\text {Follow-up }}-\mathrm{TTL}_{\mathrm{Base}}\right.$ line) was negative and significantly lower in the 
therapy compared to the control group $\left(\Delta \mathrm{TTL}_{\text {Therapy }}-\right.$ $1.00 \pm 0.53$ vs. $\left.\Delta \mathrm{TTL}_{\text {Control }} 0.85 \pm 1.21 ; p<0.001\right)$. The metabolic tumor volume increased in the control group (from $217.9 \pm 145.8 \mathrm{~mm}^{3}$ on day 0 to $573.5 \pm 294.7 \mathrm{~mm}^{3}$ on day $7 ; p=0.008$ ), while no significant change was observed in the therapy group (from $161.1 \pm 86.5 \mathrm{~mm}^{3}$ to $211.6 \pm 139.4 \mathrm{~mm}^{3} ; p=0.308$ ). Metabolic tumor volumes on follow-up were significantly higher in the control than in the therapy group $\left(573.5 \pm 294.7 \mathrm{~mm}^{3}\right.$ vs. $\left.211.6 \pm 139.4 \mathrm{~mm}^{3} ; p=0.001\right)$. Figure 3 shows representative ${ }^{18} \mathrm{~F}-\mathrm{FDG}-\mathrm{PET} / \mathrm{CT}$ data sets from the therapy and the control group. Individual TTL values and metabolic tumor volumes at baseline and at follow-up are provided in Table 1 .

\section{MRI}

DW-MRI detected an increase in ADC under therapy with a trend toward significance $\left(\mathrm{ADC}_{\text {Therapy }}\right.$ : from 0.78 $\pm 0.10 \times 10^{-3} \mathrm{~mm}^{2} / \mathrm{s}$ to $0.90 \pm 0.13 \times 10^{-3} \mathrm{~mm}^{2} / \mathrm{s} ; p=$ $0.026)$. In the control group, the ADC showed a significant decline $\left(\mathrm{ADC}_{\text {Control }}\right.$ : from $0.79 \pm 0.10 \times 10^{-3} \mathrm{~mm}^{2} / \mathrm{s}$ to $0.68 \pm 0.06 \times 10^{-3} \mathrm{~mm}^{2} / \mathrm{s} ; p=0.012$ ). Follow-up ADC values were significantly higher in the therapy group $\left(0.90 \pm 0.13 \times 10^{-3} \mathrm{~mm}^{2} / \mathrm{s}\right.$ vs. $0.68 \pm 0.06 \times 10^{-3} \mathrm{~mm}^{2} / \mathrm{s}$ in the control group; $p<0.001)$. Moreover, $\triangle \mathrm{ADC}\left(\mathrm{ADC}_{\mathrm{Fol}}\right.$ low-up $\left.-\mathrm{ADC}_{\text {Baseline }}\right)$ was significantly elevated in the therapy group $\left(\triangle \mathrm{ADC}_{\text {Therapy }} 0.12 \pm 0.14 \times 10^{-3} \mathrm{~mm}^{2} / \mathrm{s}\right.$; $\left.\Delta \mathrm{ADC}_{\text {Control }}-0.12 \pm 0.06 \times 10^{-3} \mathrm{~mm}^{2} / \mathrm{s} ; p<0.001\right)$. Analogously to the metabolic tumor volumes, the morphological tumor volumes measured on $\mathrm{T} 2 \mathrm{w}$ images significantly increased in the control group (from $134.3 \pm 89.0 \mathrm{~mm}^{3}$ to $\left.381.9 \pm 179.4 \mathrm{~mm}^{3} ; p=0.008\right)$. In the therapy group, the morphological tumor volumes showed a non-significant increase between baseline and follow-up (from $94.6 \pm$ $71.4 \mathrm{~mm}^{3}$ to $130.8 \pm 91.3 \mathrm{~mm}^{3} ; p=0.071$ ). Figure 4 shows representative MRI data sets from the therapy and the control group. Table 2 displays individual ADC values and the morphological tumor volumes for the therapy and the control group. Figure 5 shows boxplot diagrams of $\triangle T T L$ and $\triangle \mathrm{ADC}$ for the therapy and the control group.

\section{Immunohistochemistry}

Multiparametric immunohistochemistry revealed a significantly lower microvascular density (CD31: $147 \pm 48$ vs. $287 \pm 92 ; p=0.001$ ) and tumor cell proliferation (Ki67: $3718 \pm 998$ vs. $5389 \pm 1332$; $p=0.007$ ) in the therapy compared to the control group. Table 3 summarizes individual immunohistochemical values for both groups. Figure 6 shows representative tumor sections from the therapy and the control group.

\section{Correlations between imaging and immunohistochemical parameters}

TTL demonstrated a good, significant correlation to microvascular density (CD31, $\rho=0.79 ; p<0.001)$ and no significant correlation to the proliferation rate $(\mathrm{Ki}$ $67, \rho=0.33 ; p=0.14$ ). ADC showed a strong and highly significant inverse correlation to microvascular density (CD31, $\rho=-0.80 ; p<0.001$ ) but no significant correlation to proliferation (Ki-67, $\rho=-0.42 ; p=0.061$ ).

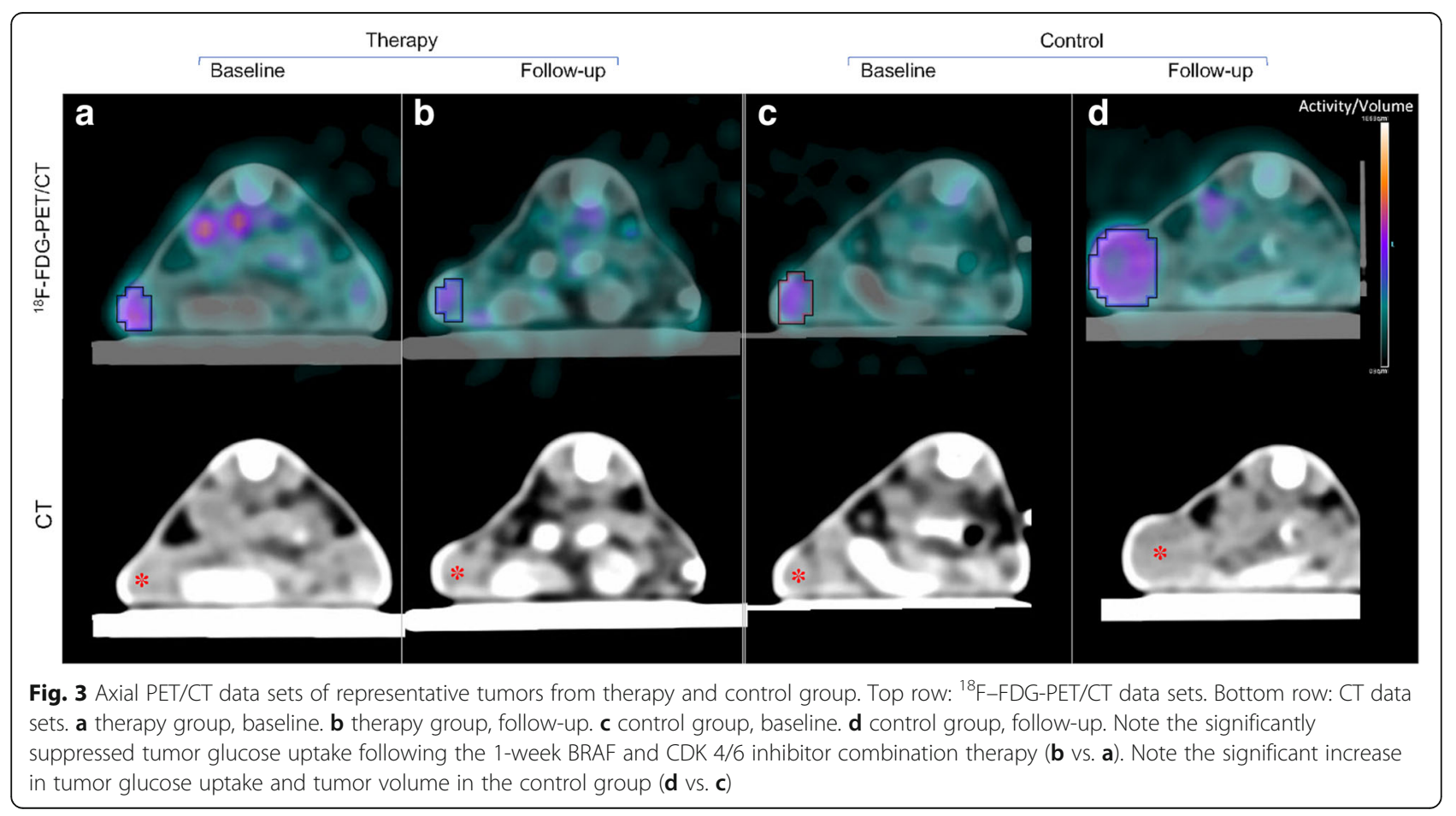


Table 1 Individual values for tumor glucose uptake and metabolic tumor volumes at baseline and follow-up

\begin{tabular}{|c|c|c|c|c|c|c|c|}
\hline Number & Group $^{a}$ & $T T L_{\text {Baseline }}$ & $T T L_{\text {Follow-Up }}$ & $\Delta T T L$ & Volume $_{\text {Baseline }}\left(\mathrm{mm}^{3}\right)$ & Volume $_{\text {Follow-up }}\left(\mathrm{mm}^{3}\right)$ & $\Delta$ Volume $\left(\mathrm{mm}^{3}\right)$ \\
\hline 1 & $\mathrm{~T}$ & 5.63 & 4.83 & -0.79 & 296.0 & 311.4 & 15.4 \\
\hline 2 & T & 4.80 & 3.13 & -1.67 & 86.8 & 50.4 & -36.4 \\
\hline 3 & T & 5.90 & 5.26 & -0.64 & 56.1 & 162.7 & 106.6 \\
\hline 4 & T & 3.72 & 2.65 & -1.07 & 155.9 & 417.9 & 262.0 \\
\hline 5 & T & 3.94 & 3.88 & 0.06 & 142.5 & 433.7 & 291.2 \\
\hline 6 & T & 4.57 & 3.02 & -1.55 & 230.8 & 185.2 & -45.6 \\
\hline 7 & T & 4.61 & 2.90 & -1.71 & 112.8 & 78.2 & -34.6 \\
\hline 8 & T & 3.69 & 2.40 & -1.29 & 162.7 & 118.0 & -44.7 \\
\hline 9 & T & 3.11 & 2.52 & -0.59 & 332.0 & 392.0 & 60.0 \\
\hline 10 & T & 3.59 & 2.46 & -1.13 & 171.3 & 161.7 & -9.6 \\
\hline 11 & T & 4.14 & 2.93 & -1.21 & 120.0 & 80.6 & -39.4 \\
\hline 12 & T & 2.57 & 2.29 & -0.28 & 66.2 & 147.8 & 81.6 \\
\hline Mean $\pm S D^{b}$ & T & $4.19 \pm 0.97$ & $3.19 \pm 0.97$ & $-1.00 \pm 0.53$ & $161.1 \pm 86.5$ & $211.6 \pm 139.4$ & $50.5 \pm 117.9$ \\
\hline 13 & C & 4.34 & 4.06 & -0.28 & 96.9 & 344.0 & 247.1 \\
\hline 14 & C & 3.07 & 5.13 & 2.06 & 519.1 & 1192.3 & 673.2 \\
\hline 15 & $C$ & 5.62 & 5.50 & -0.12 & 213.0 & 616.1 & 403.1 \\
\hline 16 & $C$ & 2.88 & 3.95 & 1.07 & 140.1 & 329.1 & 189.0 \\
\hline 17 & $C$ & 4.02 & 4.13 & 0.11 & 222.6 & 597.8 & 375.2 \\
\hline 18 & $C$ & 3.31 & 5.25 & 1.94 & 165.1 & 511.0 & 345.9 \\
\hline 19 & $C$ & 3.50 & 5.45 & 1.95 & 115.6 & 421.3 & 305.7 \\
\hline 20 & C & 2.71 & 4.73 & 2.02 & 392.5 & 866.5 & 474.0 \\
\hline 21 & C & 3.81 & 2.72 & -1.09 & 96.0 & 283.1 & 187.1 \\
\hline Mean $\pm S D^{b}$ & C & $3.70 \pm 0.90$ & $4.55 \pm 0.91$ & $0.85 \pm 1.21$ & $217.9 \pm 145.8$ & $573.5 \pm 294.7$ & $355.6 \pm 153.3$ \\
\hline
\end{tabular}

${ }^{\mathrm{a}} \mathrm{T}=$ therapy group; $\mathrm{C}=$ control group

${ }^{\mathrm{b}} \mathrm{SD}=$ standard deviation

$\triangle \mathrm{TTL}$ and $\triangle \mathrm{ADC}$ showed strong and significant inverse correlations $(\rho=-0.75 ; p=0.002)$.

\section{Discussion}

In the present study, we investigated ${ }^{18} \mathrm{~F}-\mathrm{FDG}-\mathrm{PET} / \mathrm{CT}$ and DW-MRI for the in vivo monitoring of a novel BRAF and CDK4/6 inhibitor combination therapy in human melanoma xenografts in mice. Dual inhibition of the MAPK signal pathway demonstrated significant antiangiogenic and anti-proliferative effects in the investigated tumor model. The multimodal imaging protocol allowed for the in vivo monitoring of tumor glucose metabolism and tumor diffusivity, adding molecular and functional information to the established morphologybased assessments of tumor response.

Our results are in line with previous preclinical and clinical studies investigating tumor response to targeted MAPK signal pathway inhibition. Baudy et al. reported a reduction in tumor glucose metabolism in A375 xenografts in mice following a BRAF (vemurafenib) and MEK inhibitor (GDC-0973) combination therapy over the course of 6 days [21]. However, the authors validated the imaging results by tumor cell glucose transporter 1 and MAPK pathway protein expression but not by immunohistochemical markers of microvascular density or tumor cell proliferation. Analogously, combined BRAF and MEK targeting (vemurafenib plus cobimetinib or dabrafenib plus trametinib) lead to a significant reduction in tumor maximum standardized uptake value (SUVmax) in patients with advanced melanoma with a mean time to follow-up of 26 days [22]. ${ }^{18}$ F-FDG-PET even provided predictive imaging biomarkers of therapy response in the investigated patient population, with a significant association of the change in SUVmax and progression-free survival observed for the least responsive tumor focus [22]. These studies underline the applicability and clinical significance of ${ }^{18} \mathrm{~F}-\mathrm{FDG}$-based hybrid imaging for therapy monitoring in melanoma under MAPK pathway inhibition. Providing a surrogate of tumor cellularity, DW-MRI confirmed the ${ }^{18} \mathrm{~F}-\mathrm{FDG}$ PET results and may thus be a suitable imaging modality to allow for a multi-facetted tumor characterization under targeted therapy. In experimental human BRAFmutant melanomas, DW-MRI was successfully used for 


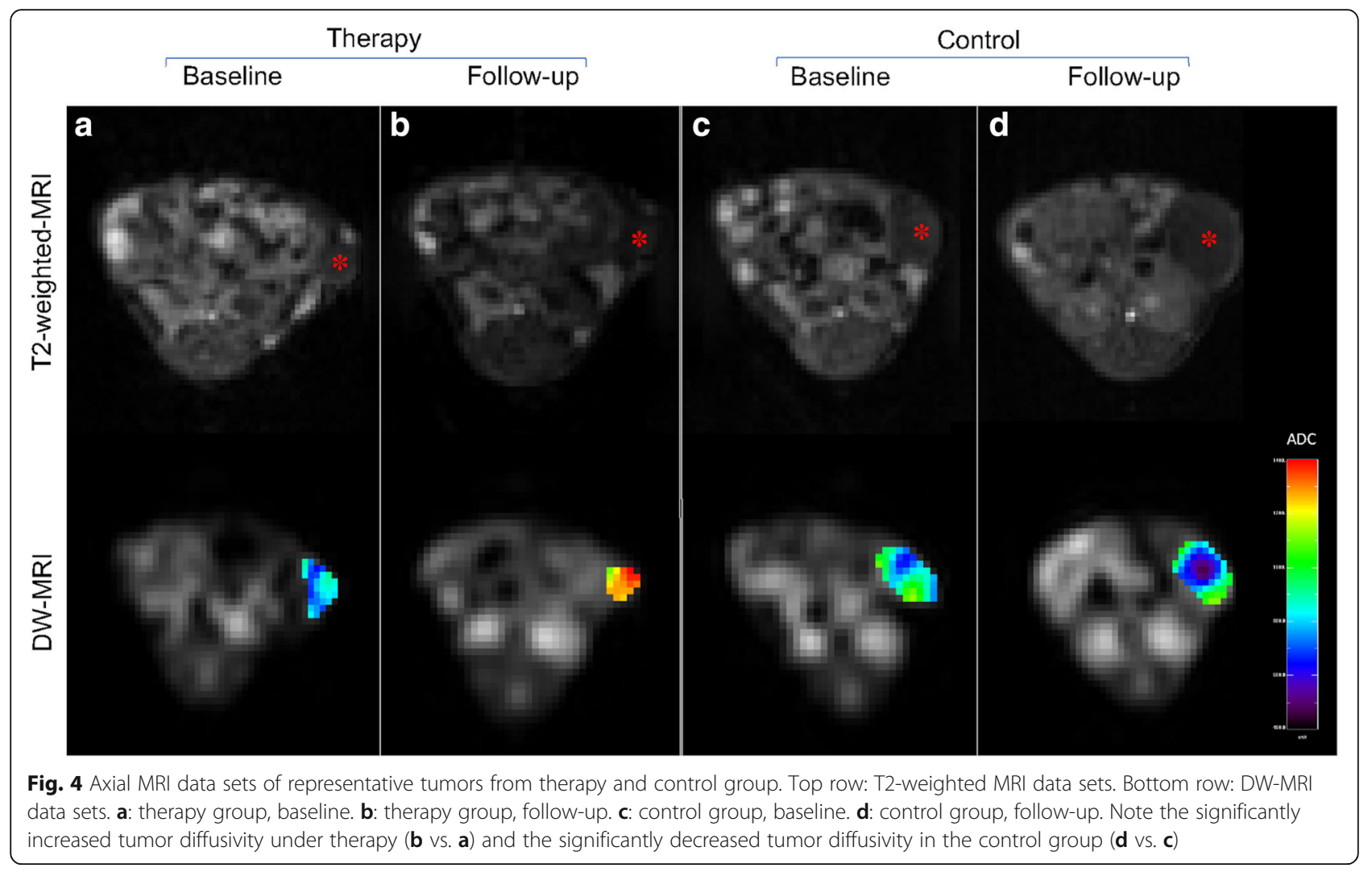

the in vivo monitoring of a 4-day therapy with the MEK inhibitor selumetinib [17]. Our results confirm the applicability of both ${ }^{18}$ F-FDG-PET and DW-MRI for therapy monitoring in the same tumors showing a strong, intraindividual correlation between $\triangle \mathrm{TTL}$ and $\triangle \mathrm{ADC}$. This correlation may be explained by the fact that a high tumor cell density leads to restricted diffusion with low ADC values and increased tumor glucose metabolism [23].

The addition of a MEK inhibitor to BRAF inhibitor monotherapy proved to be a successful strategy to overcome intrinsic or de novo BRAF inhibitor resistance in BRAF-mutant melanoma [24]. However, resistance was also reported for the BRAF/MEK inhibitor combination therapy [25]. Therefore, novel compounds to be used in combination with BRAF inhibitors are required to address this clinical challenge and need to be investigated in experimental and clinical studies. As a proof of principle, the present study is a first approach to close this gap of knowledge, exploring a selective CDK 4/6 inhibitor as novel combination compound for dual inhibition of the MAPK signal pathway. Our preclinical results add to the literature providing first evidence that a novel BRAF and CDK 4/6 inhibitor combination therapy may be an effective therapeutic regimen in BRAFmutant melanoma, with significant effects on tumor glucose metabolism, tumor diffusivity, microvascular density, and tumor cell proliferation. However, these promising preclinical results require further validation in future clinical studies. Selective CDK 4/6 inhibition was shown to significantly improve progression-free survival in patients with advanced breast cancer, but to date there are no clinical data on combined BRAF and CDK 4/6 inhibition $[11,26]$. Our results indicate that ${ }^{18}$ F-FDG-PET/CT and DW-MRI may be applied for non-invasive therapy guidance in clinical trials investigating targeted MAPK pathway inhibition, adding functional and molecular biomarkers of therapy response to morphology-based response criteria. Measurements of tumor radiotracer uptake and ADC can easily be implemented in clinical routine staging reports. Clinically, ${ }^{18}$ F-FDG-PET/CT is widely applied for staging in advanced melanoma. The current European Society for Medical Oncology guidelines recommend $\mathrm{CT}$ or ${ }^{18} \mathrm{~F}$-FDG-PET in advanced cutaneous melanoma (> pT3a) prior to surgical treatment and sentinel lymph node biopsy [27]. The National Comprehensive Cancer Network Clinical Practice Guidelines in Oncology for Melanoma Version 01.2017 recommend CT and/or ${ }^{18}$ F-FDG-PET for treatment response assessment in patients receiving active non-surgical treatment (www.nccn.org). While ${ }^{18} \mathrm{~F}-\mathrm{FDG}-\mathrm{PET} / \mathrm{CT}$ provides a comprehensive wholebody tumor staging at a high spatial resolution, MRI is mainly applied for the local staging of defined body 
Table 2 Individual values for tumor diffusivity and morphological tumor volumes at baseline and follow-up

\begin{tabular}{|c|c|c|c|c|c|c|c|}
\hline Number & Group $^{a}$ & $\operatorname{ADC}_{\text {Baseline }}\left(\mathrm{mm}^{2} \times 10^{-3} / \mathrm{s}\right)$ & $A D C_{\text {Follow-up }}\left(\mathrm{mm}^{2} \times 10^{-3} / \mathrm{s}\right)$ & $\triangle \mathrm{ADC}\left(\mathrm{mm}^{2} \times 10^{-3} / \mathrm{s}\right)$ & Volume $_{\text {Baseline }}\left(\mathrm{mm}^{3}\right)$ & Volume $_{\text {Follow-up }}\left(\mathrm{mm}^{3}\right)$ & $\Delta$ Volume $\left(\mathrm{mm}^{3}\right)$ \\
\hline 1 & $T$ & 0.84 & 0.84 & 0.00 & 163.0 & 166.3 & 3.3 \\
\hline 2 & T & 0.88 & 1.16 & 0.28 & 25.7 & 33.5 & 7.8 \\
\hline 3 & T & 0.74 & 0.72 & -0.02 & 19.1 & 99.5 & 80.4 \\
\hline 4 & T & 0.77 & 0.86 & 0.09 & 188.8 & 255.5 & 66.7 \\
\hline 5 & T & 0.87 & 0.82 & -0.05 & 88.5 & 281.1 & 192.6 \\
\hline 6 & T & 0.62 & 0.84 & 0.22 & 154.9 & 107.7 & -47.2 \\
\hline 7 & T & 0.79 & 1.07 & 0.28 & 67.2 & 48.3 & -18.9 \\
\hline 8 & $\mathrm{~T}$ & 0.71 & 0.87 & 0.16 & 38.7 & 50.8 & 12.1 \\
\hline 9 & T & 0.66 & 0.83 & 0.17 & 232.5 & 264.1 & 31.6 \\
\hline 10 & T & 0.73 & 0.81 & 0.08 & 62.0 & 123.0 & 61.0 \\
\hline 11 & T & 0.76 & 1.07 & 0.31 & 48.9 & 31.9 & -17.0 \\
\hline 12 & T & 0.97 & 0.85 & -0.12 & 45.6 & 107.9 & 62.3 \\
\hline Mean $\pm S D^{b}$ & $\mathrm{~T}$ & $0.78 \pm 0.10$ & $0.90 \pm 0.13$ & $0.12 \pm 0.14$ & $94.6 \pm 71.4$ & $130.8 \pm 91.3$ & $36.2 \pm 63.2$ \\
\hline 13 & C & 0.85 & 0.77 & -0.08 & 119.0 & 356.0 & 237.0 \\
\hline 14 & C & 0.70 & 0.63 & -0.07 & 320.6 & 777.6 & 457.0 \\
\hline 15 & C & 0.86 & 0.69 & -0.17 & 103.1 & 379.7 & 276.6 \\
\hline 16 & C & 0.96 & 0.76 & -0.20 & 81.5 & 212.9 & 131.4 \\
\hline 17 & C & 0.78 & 0.63 & -0.15 & 138.0 & 371.4 & 233.4 \\
\hline 18 & C & 0.73 & 0.61 & -0.12 & 109.0 & 292.9 & 183.9 \\
\hline 19 & C & 0.87 & 0.71 & -0.16 & 72.7 & 301.7 & 229.0 \\
\hline 20 & C & 0.61 & 0.61 & 0.00 & 232.7 & 541.0 & 308.3 \\
\hline 21 & C & 0.79 & 0.69 & -0.10 & 31.8 & 203.5 & 171.7 \\
\hline Mean $\pm S D^{b}$ & c & $0.79 \pm 0.10$ & $0.68 \pm 0.06$ & $-0.12 \pm 0.06$ & $134.3 \pm 89.0$ & $381.9 \pm 179.4$ & $247.6 \pm 95.1$ \\
\hline
\end{tabular}

${ }^{\mathrm{a}} \mathrm{T}=$ therapy group; $\mathrm{C}=$ control group

${ }^{\mathrm{b}} \mathrm{SD}=$ standard deviation

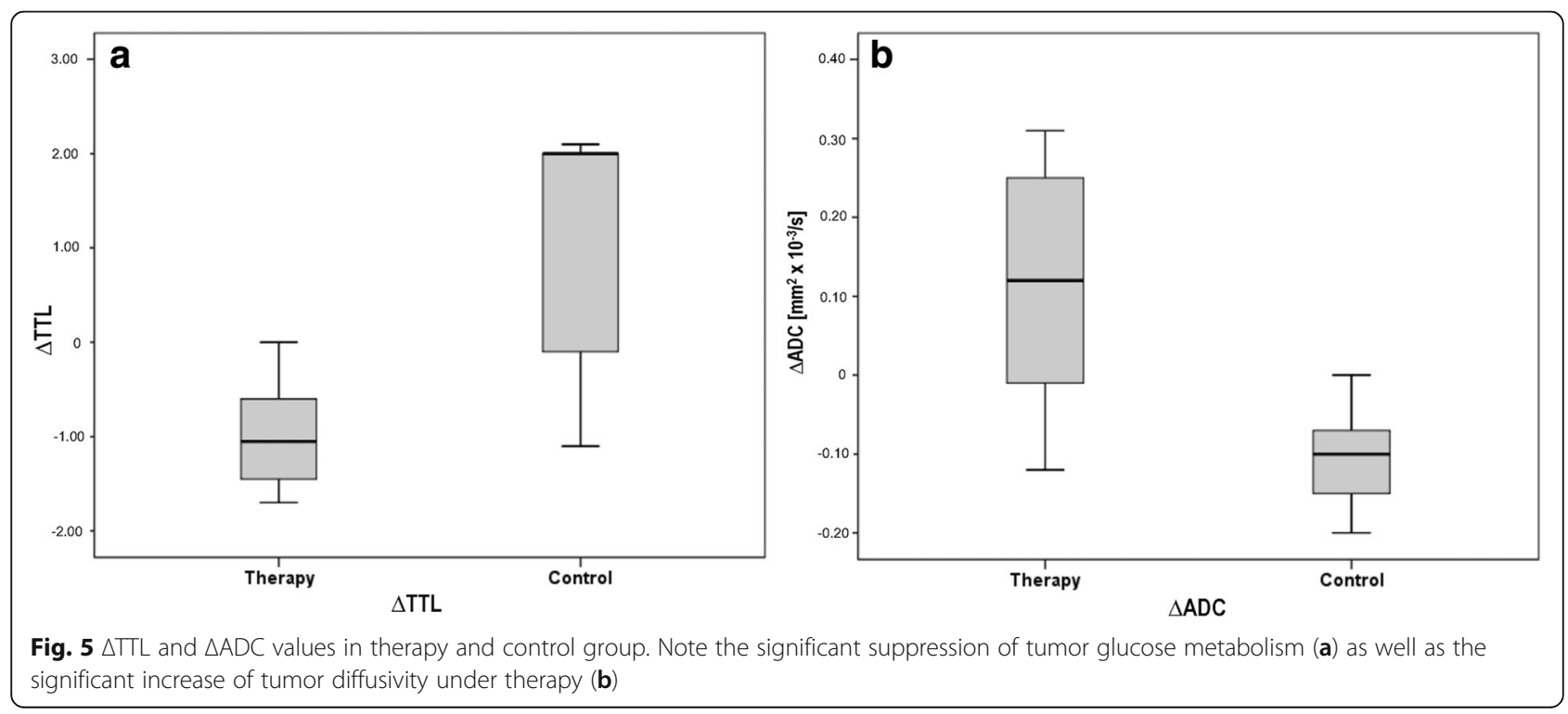


Table 3 Individual immunohistochemical parameters

\begin{tabular}{|c|c|c|c|}
\hline Number & Group $^{a}$ & CD31 & Ki-67 \\
\hline 1 & $\mathrm{~T}$ & 166 & 4433 \\
\hline 2 & $\mathrm{~T}$ & 83 & 5831 \\
\hline 3 & T & 212 & 3189 \\
\hline 4 & $\mathrm{~T}$ & 129 & 2649 \\
\hline 5 & $\mathrm{~T}$ & 222 & 2832 \\
\hline 6 & $\mathrm{~T}$ & 182 & 3820 \\
\hline 7 & $\mathrm{~T}$ & 79 & 4033 \\
\hline 8 & $\mathrm{~T}$ & 105 & 2912 \\
\hline 9 & T & 100 & 2227 \\
\hline 10 & T & 151 & 4306 \\
\hline 11 & T & 179 & 4210 \\
\hline 12 & T & 151 & 4177 \\
\hline Mean $\pm S D^{b}$ & $\mathrm{~T}$ & $147 \pm 48$ & $3718 \pm 998$ \\
\hline 13 & C & 271 & 6801 \\
\hline 14 & C & 336 & 6349 \\
\hline 15 & C & 447 & 6489 \\
\hline 16 & C & 191 & 3799 \\
\hline 17 & C & 307 & 4562 \\
\hline 18 & C & 358 & 4582 \\
\hline 19 & C & 261 & 3379 \\
\hline 20 & C & 284 & 5718 \\
\hline 21 & C & 132 & 6824 \\
\hline Mean $\pm S D^{b}$ & C & $287 \pm 92$ & $5389 \pm 1332$ \\
\hline
\end{tabular}

${ }^{\mathrm{a}} \mathrm{T}=$ therapy group; $\mathrm{C}=$ control group

${ }^{\mathrm{b}} \mathrm{SD}=$ standard deviation regions, e. g., for the detection of brain and liver metastases, and may therefore complement whole-body imaging protocols for the longitudinal assessment of therapy response [28]. State-of-the-art DW-MRI sequences, e. g., for the brain or abdomen, can be acquired in less than 3 min and may be integrated in standard MRI protocols without significant prolongation of the acquisition time.

\section{Limitations}

The study is limited in several aspects. First, although all efforts were made to maintain the same scanning position between PET and MRI as well as between baseline and follow-up, it cannot be fully excluded that the animals were scanned in slightly different planes with potential effects on the semiquantitative parameters. Hybrid imaging on an integrated PET/MRI scanner with simultaneous acquisition of both modalities may improve consistency and concordance of the PET and MRI results due to equalized scanning conditions. Second, ex vivo immunohistochemical analysis was performed in selected cross-sections of the tumor and may not necessarily correspond to the whole-tumor VOI. Third, we investigated the BRAF and CDK 4/6 inhibitor combination therapy over the course of 1 week but did not compare the combination therapy to the BRAF inhibitor monotherapy. The comparison of the different therapy regimens in the same tumor model and a longer follow-up interval may provide additional insights in tumor pathophysiology and time course of acquired resistance under MAPK pathway inhibition. Fourth, due to study design, immunohistochemical parameters were only acquired on day 7 after follow-up imaging.

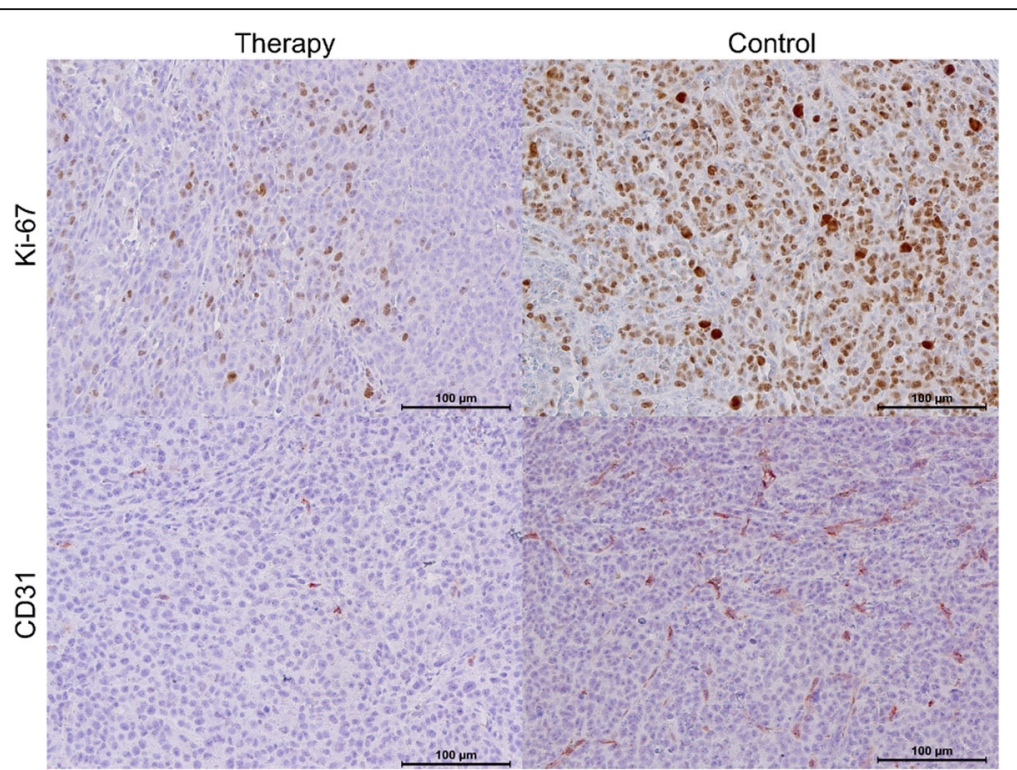

Fig. 6 Tumor cell proliferation and microvascular density in representative tumor sections. Left column: therapy. Right column: control. Top row: proliferation (Ki-67). Bottom row: microvascular density (CD31). Note the significantly lower tumor proliferation (Ki-67) and tumor microvascular density (CD31) in the therapy compared to the control group 


\section{Conclusion}

In conclusion, we demonstrated that a novel BRAF and CDK 4/6 inhibitor combination therapy exhibits significant anti-angiogenic and anti-proliferative effects in experimental human melanomas in mice and that the according alterations in tumor pathophysiology can be monitored non-invasively and in vivo by ${ }^{18} \mathrm{~F}-\mathrm{FDG}$-PET/ CT and DW-MRI. Co-targeting the MAPK pathway using a BRAF and CDK 4/6 inhibitor combination therapy is a novel approach to potentially overcome intrinsic or de novo BRAF inhibitor resistance in BRAF-mutant melanoma and may be investigated in future clinical trials.

\begin{abstract}
Abbreviations
${ }^{18} \mathrm{~F}-\mathrm{FDG}$-PET: ${ }^{18} \mathrm{~F}$-Fluorodeoxyglucose positron emission tomography; ADC: Apparent diffusion coefficient; BRAF: B-rapidly accelerated fibrosarcoma; CDK: Cyclin-dependent kinase; CT: Computed tomography; DW-MRI: Diffusionweighted magnetic resonance imaging; MAPK: Mitogen-activated protein kinase; MEK: Mitogen-activated extracellular signal-regulated kinase; MTV: Metabolic tumor volume; PMI: Platform for research in medical imaging; RECIST: Response evaluation criteria in solid tumors; SUV: Standardized uptake value; TTL: Tumor-toLiver-Ratio; VOI: Volume of interest
\end{abstract}

\section{Acknowledgements}

We want to acknowledge the contribution of Rosel Oos from the Department of Nuclear Medicine (University Hospital, Ludwig-MaximiliansUniversity Munich) for her extensive technical support.

\section{Funding}

This study was supported by a research grant from Novartis AG (Basel, Switzerland). The therapeutics (dabrafenib and ribociclib; Novartis AG, Basel, Switzerland) were supplied by the manufacturer free of charge.

\section{Availability of data and materials}

All data generated or analysed during this study are included in this published article.

\section{Authors' contributions}

RSE, PMK, AT, CCC, PB, MFR and JR were major contributors in the conception and writing of the manuscript. RSE, PMK, MMH, GK, MJS, WGK and OS performed the MRI measurements. RSE, PMK, AT, MMH, WGK, OS and JO performed the PET/CT measurements. RSE, PMK, CCC, AT, MMH, MJS, PB, MFR and JR analyzed and interpreted the imaging data, performed the postprocessing and the statistical analysis. $\mathrm{HH}-\mathrm{E}, \mathrm{MMH}, \mathrm{WGK}, \mathrm{GK}, \mathrm{JO}$ and OS performed the immunohistological examinations of the tumors and the analysis of the stainings. $\mathrm{HH}-\mathrm{E}, \mathrm{RSE}, \mathrm{PMK}, \mathrm{MMH}, \mathrm{OS}$ and $\mathrm{GK}$ administered the daily medication. All authors did substantial contributions to the conception of the work, or the acquisition, analysis, or interpretation of the data. All authors agreed to be accountable for all aspects of the work, read and revised the draft critically and approved the final manuscript.

\section{Ethics approval}

All experiments were performed in strict accordance with the Guidelines for the Care and Use of Laboratory Animals of the National Institutes of Health and with approval by the Government of Upper Bavaria Committee for Animal Research (Gz. 55.2-1-54-2532-36-2016).

\section{Consent for publication}

Not applicable.

\section{Competing interests}

CCC: Speakers' bureau, Siemens Healthineers. All other authors declare that they have no competing interests.

\section{Publisher's Note}

Springer Nature remains neutral with regard to jurisdictional claims in published maps and institutional affiliations.

\section{Author details}

${ }^{1}$ Department of Radiology, Laboratory for Experimental Radiology, University Hospital, Ludwig-Maximilians-University Munich, Marchioninistr. 15, 81377 München, Germany. ${ }^{2}$ Department of Nuclear Medicine, University Hospital, Ludwig-Maximilians-University Munich, Marchioninistr. 15, 81377 München, Germany. ${ }^{3}$ Comprehensive Pneumology Center, German Center for Lung Research, Munich, Germany.

Received: 22 November 2017 Accepted: 5 January 2018

Published online: 18 January 2018

\section{References}

1. Amaral T, Sinnberg T, Meier F, Krepler C, Levesque M, Niessner $H$, et al. The mitogen-activated protein kinase pathway in melanoma part I - activation and primary resistance mechanisms to BRAF inhibition. Eur J Cancer. 2017;73:85-92.

2. Yadav V, Chen SH, Yue YG, Buchanan S, Beckmann RP, Peng SB. Cotargeting BRAF and cyclin dependent kinases 4/6 for BRAF mutant cancers. Pharmacol Ther 2015;149:139-149.

3. Hertzman Johansson C, Egyhazi BS. BRAF inhibitors in cancer therapy. Pharmacol Ther. 2014;142:176-82.

4. Chmielowski B. Is there a role for single-agent BRAF inhibition in melanoma? Clin Adv Hematol Oncol. 2017;15:108-10.

5. Manzano JL, Layos L, Buges C, de Los Llanos Gil M, Vila L, Martinez-Balibrea E, et al. Resistant mechanisms to BRAF inhibitors in melanoma. Ann Transl Med. 2016;4:237.

6. Sun C, Wang L, Huang S, Heynen GJ, Prahallad A, Robert C, et al. Reversible and adaptive resistance to BRAF(V600E) inhibition in melanoma. Nature. 2014;508:118-22.

7. Villanueva J, Vultur A, Herlyn M. Resistance to BRAF inhibitors: unraveling mechanisms and future treatment options. Cancer Res. 2011;71:7137-40.

8. Robert C, Karaszewska B, Schachter J, Rutkowski P, Mackiewicz A, Stroiakovski D, et al. Improved overall survival in melanoma with combined dabrafenib and trametinib. N Engl J Med. 2015;372:30-9.

9. da Silveira Nogueira Lima JP, Georgieva M, Haaland B, de Lima Lopes G. A systematic review and network meta-analysis of immunotherapy and targeted therapy for advanced melanoma. Cancer Med. 2017;6(6):1143-53.

10. Yadav V, Burke TF, Huber L, Van Horn RD, Zhang Y, Buchanan SG, et al. The CDK4/6 inhibitor LY2835219 overcomes vemurafenib resistance resulting from MAPK reactivation and cyclin D1 upregulation. Mol Cancer Ther. 2014; 13:2253-63.

11. Hortobagyi GN, Stemmer SM, Burris HA, Yap YS, Sonke GS, Paluch-Shimon S, et al. Ribociclib as first-line therapy for HR-positive, advanced breast cancer. N Engl J Med. 2016;375:1738-48.

12. Eisenhauer EA, Therasse P, Bogaerts J, Schwartz LH, Sargent D, Ford R, et al. New response evaluation criteria in solid tumours: revised RECIST guideline (version 1.1). Eur J Cancer. 2009;45:228-47.

13. Desar IM, van Herpen CM, van Laarhoven HW, Barentsz JO, Oyen WJ, van der Graaf WT. Beyond RECIST: molecular and functional imaging techniques for evaluation of response to targeted therapy. Cancer Treat Rev. 2009;35:309-21.

14. Teng FF, Meng X, Sun XD, Yu JM. New strategy for monitoring targeted therapy: molecular imaging. Int J Nanomedicine. 2013;8:3703-13.

15. McArthur GA, Puzanov I, Amaravadi R, Ribas A, Chapman P, Kim KB, et al. Marked, homogeneous, and early [18F]fluorodeoxyglucose-positron emission tomography responses to vemurafenib in BRAF-mutant advanced melanoma. J Clin Oncol. 2012;30:1628-34.

16. Geven EJ, Evers S, Nayak TK, Bergstrom M, Su F, Gerrits D, et al. Therapy response monitoring of the early effects of a new BRAF inhibitor on melanoma xenograft in mice: evaluation of (18) F-FDG-PET and (18) F-FLTPET. Contrast Media Mol Imaging. 2015;10:203-10.

17. Beloueche-Babari M, Jamin Y, Arunan V, Walker-Samuel S, Revill M, Smith $P D$, et al. Acute tumour response to the MEK1/2 inhibitor selumetinib (AZD6244, ARRY-142886) evaluated by non-invasive diffusion-weighted MRI. Br J Cancer. 2013;109:1562-9.

18. Gaustad JV, Pozdniakova V, Hompland T, Simonsen TG, Rofstad EK. Magnetic resonance imaging identifies early effects of sunitinib treatment in human melanoma xenografts. J Exp Clin Cancer Res. 2013;32:93.

19. Kazmierczak PM, Todica A, Gildehaus FJ, Hirner-Eppeneder H, Brendel M, Eschbach RS, et al. 68Ga-TRAP-(RGD)3 hybrid imaging for the in vivo monitoring of alphavss3-Integrin expression as biomarker of antiAngiogenic therapy effects in experimental breast cancer. PLoS One. 2016; $11: e 0168248$. 
20. Sourbron S, Ingrisch M, Siefert A, Reiser M, Herrmann K. Quantification of cerebral blood flow, cerebral blood volume, and blood-brain-barrier leakage with DCE-MRI. Magn Reson Med. 2009;62:205-17.

21. Baudy AR, Dogan T, Flores-Mercado JE, Hoeflich KP, Su F, van Bruggen N, et al. FDG-PET is a good biomarker of both early response and acquired resistance in BRAFV600 mutant melanomas treated with vemurafenib and the MEK inhibitor GDC-0973. EJNMMI Res. 2012;2:22.

22. Schmitt RJ, Kreidler SM, Glueck DH, Amaria RN, Gonzalez R, Lewis K, et al. Correlation between early 18F-FDG PET/CT response to BRAF and MEK inhibition and survival in patients with BRAF-mutant metastatic melanoma. Nucl Med Commun. 2016;37:122-8.

23. Surov A, Meyer HJ, Wienke A. Correlation between minimum apparent diffusion coefficient (ADCmin) and tumor Cellularity: a meta-analysis. Anticancer Res. 2017;37:3807-10.

24. Flaherty KT, Infante JR, Daud A, Gonzalez R, Kefford RF, Sosman J, et al. Combined BRAF and MEK inhibition in melanoma with BRAF V600 mutations. N Engl J Med. 2012;367:1694-703.

25. Wagle N, Van Allen EM, Treacy DJ, Frederick DT, Cooper ZA, Taylor-Weiner A, et al. MAP kinase pathway alterations in BRAF-mutant melanoma patients with acquired resistance to combined RAF/MEK inhibition. Cancer Discov. 2014;:4:61-8.

26. Finn RS, Crown JP, Lang I, Boer K, Bondarenko IM, Kulyk SO, et al. The cyclin-dependent kinase $4 / 6$ inhibitor palbociclib in combination with letrozole versus letrozole alone as first-line treatment of oestrogen receptorpositive, HER2-negative, advanced breast cancer (PALOMA-1/TRIO-18): a randomised phase 2 study. Lancet Oncol. 2015;16:25-35.

27. Dummer R, Hauschild A, Lindenblatt N, Pentheroudakis G, Keilholz U, Committee EG. Cutaneous melanoma: ESMO clinical practice guidelines for diagnosis, treatment and follow-up. Ann Oncol. 2015;26(Suppl 5):v126-32.

28. Perng P, Marcus C, Subramaniam RM. (18)F-FDG PET/CT and melanoma: staging, immune modulation and mutation-targeted therapy assessment, and prognosis. AJR Am J Roentgenol. 2015;205:259-70.

\section{Submit your next manuscript to BioMed Central and we will help you at every step:}

- We accept pre-submission inquiries

- Our selector tool helps you to find the most relevant journal

- We provide round the clock customer support

- Convenient online submission

- Thorough peer review

- Inclusion in PubMed and all major indexing services

- Maximum visibility for your research

Submit your manuscript at www.biomedcentral.com/submit 